\title{
Answer to comment on the paper of S. Dorbolo et al., Physica C 267 (1996) 24-30 entitled: Influence of Van Hove singularity on the electronic specific heat of high- $T_{\mathrm{c}}$ superconductors
}

\author{
S. Dorbolo * \\ S.U.P.R.A.S Institut de Physique B5, Université de Liège, B-4000 Liège, Belgium
}

Received 30 December 1996

In their comment [1], J. Bok and J. Bouvier mentioned the lack of validity of work starting with the specific heat formula Eq. (1) in our paper [2]. They discussed two other points i.e. the variation of the physical parameters as well as the electronic band structure. Even though their points are well taken, we show that our conclusions are still valid from a qualitative point of view.

In thermodynamics, the specific heat can be considered as the first derivative of the entropy, the first derivative of the internal energy or the second derivative of the free energy [3]. In Ref. [4], G. Rickayzen starts from the entropy $S$ in order to compute the electronic specific heat

$C_{\mathrm{e}}=T\left(\frac{\partial S}{\partial T}\right)_{V}=\sum_{k} E_{k} \frac{\partial f_{k}}{\partial T}$

where $f_{k}$ is the Fermi-Dirac distribution function and $E_{k}$ is the quasiparticle spectrum: $E_{k}^{2}=(\epsilon(k)-$ $\left.\epsilon_{\mathrm{F}}\right)^{2}+\Delta^{2}$.

We computed the electronic specific heat $C_{\mathrm{e}}$ from

$C_{\mathrm{e}}=\left(\frac{\partial U}{\partial T}\right)_{V}=\frac{\partial}{\partial T}\left(\sum_{k} E_{k} f_{k}\right)$

\footnotetext{
*E-mail: dorbolo@gw.unipc.ulg.ac.be.
}

with $U$ the internal energy. It is claimed in Eq. (1) that the last equality in Eq. (2) is invalid. In fact, the quasiparticle spectrum is defined by

$E_{k}=\left(\partial U / \partial f_{k}\right)_{u_{k}, v_{k}}$,

where $u_{k}$ and $v_{k}$ are the coefficients of the linear combination of creation and destruction operators of quasiparticles [5]. Therefore, after taking the temperature derivative of $U$ in Eq. (3), Eq. (1) is found again. The difference between Eq. (2) and the r.h.s of Eq. (1) is $\sum_{k}\left(\partial E_{k} / \partial T\right) f_{k}$. This term becomes the most important one close to $T_{\mathfrak{c}}$ when the gap varies strongly.

Using Eq. (1), a few modifications to our previous results occur but the main qualitative conclusions are exactly obtained.

In Fig. 1, we compare the electronic specific heat calculated with Eq. (2) and Eq. (1) for the same set of physical parameters $\Delta(0)=20 \mathrm{meV}$ and $m^{*}=8$ $m_{0}$. At low temperature, both results are quite similar. A linear contribution is observed. As expected, the deviation is more important when the temperature lies close to $T_{c}$.

The specific heat singularity at $T_{c}$ observed in many systems, though quantitatively different from each other, can still be explained with a quasiparticle spectrum containing a d-wave gap parameter for reasonable values of the physical parameters i.e. 


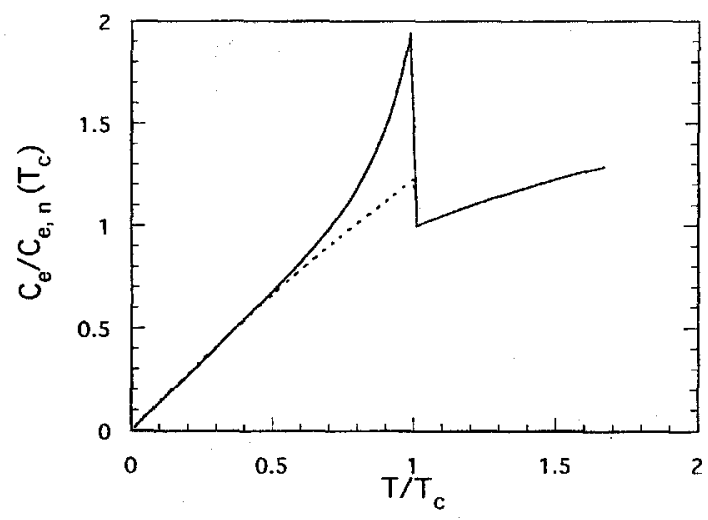

Fig. 1. Comparison of the calculation of the electronic specific heat from Eq. (1) (solid line) and from Eq. (2) (dashed line).

$\Delta(0) \approx 30 \mathrm{meV}$ and $m^{*}=8 m_{0}$ when Van Hove singularities are present in the band structure.

The temperature at which $C_{\mathrm{e}, \mathrm{s}}=C_{\mathrm{e}, \mathrm{n}}$ lies between 0.6 and $0.8 T / T_{c}$ in the s-wave case and between 0.4 and $0.6 T / T_{\mathrm{c}}$ in the d-wave case, respectively. Those results are thus quite similar to those in Ref. [2].

It is relevant to know how the value of the normalized jump $\Delta C_{\mathrm{e}, \mathrm{n}}$ varies with physical parameters such as the effective mass $m^{*}$ or the ratio $\Delta(0) / k_{\mathrm{B}} T_{\mathrm{c}}$ since the $\mathrm{BCS}$ theory gives [1]

$\Delta C_{\mathrm{e}, \mathrm{n}} \propto\left(\Delta(0) / k_{\mathrm{B}} T_{\mathrm{c}}\right)^{2}$.

When the gap parameter $\Delta(0)$ is changed without changing the critical temperature, we can point out how the electronic specific heat depends on the ratio $\Delta(0) / k_{\mathrm{B}} T_{\mathrm{c}}$. We find numerically that the normalized jump in the s-wave case including Van Hove singularities in the band structure behaves like

$$
\Delta C_{\mathrm{e}, \mathrm{n}}=1.1419\left(\Delta(0) / k_{\mathrm{B}} T_{\mathrm{c}}\right)^{1.9685} \text {. }
$$

Eq. (5) is similar to the BCS equation mentioned by Bok and Bouvier [1]. The phenomenological expression describing the normalized jump in the d-wave case is however quite different, namely

$\Delta C_{\mathrm{e}, \mathrm{n}}=0.21139\left(\Delta(0) / k_{\mathrm{B}} T_{\mathrm{c}}\right)^{1.6724}$,

where the exponent is markedely different and equals about $5 / 3$. It will be an interesting check of the Bok and Bouvier comment [1] with respect to our work [2] whether the exponent is equal to 2 or $5 / 3$.

Finally, we agree that the real electronic band structure is indeed quite complicated. Nevertheless the Hubbard model [6] gives a realistic band structure, namely

$\epsilon(k)=-2 t\left(\cos k_{x}+\cos k_{y}\right)+4 t^{\prime} \cos k_{x} \cos k_{y}$,

where $t$ and $t^{\prime}$ are the hopping integrals between nearest neighbours and between next-nearest neighbours respectively. This band structure is characterized by four saddle points at $(0, \pm \pi / b)$ and $( \pm \pi / a, 0)$. Before considering such a complicated band structure, we wanted to isolate the effect of saddle points taking place in the same directions as in the Hubbard band. Therefore we considered only the following band structure:

$$
\epsilon(k)-\epsilon_{\mathrm{F}}=\left(\hbar^{2} / 2 m^{*}\right) k_{x} k_{y} .
$$

This latter energy spectrum presents an extended saddle point along the $k_{x}=0$ and $k_{y}=0$ directions. Moreover, such a band was used in Ref. [7] and reproduces the band structure measurements of the ARPES experiment [8]. It is of course entirely true that $\epsilon_{\mathrm{F}}, m^{*}$ and $\Delta(0)$ are functions of $t, t^{\prime}, \ldots$ Typically for the fit used in Ref. [2], the physical parameters should be $t=0.40 \mathrm{eV}$, hence independent of $t^{\prime}$ [9] as can be easily checked.

In conclusion, the comments by Bok and Bouvier about the validity of the starting specific heat formula are founded. However, our qualitative conclusions are correct namely the only quasiparticle spectrum reproducing the experimental behaviour is a spectrum containing a d-wave gap parameter in order to explain the low temperature behaviour. Besides, the Van Hove singularities in the band structure allow us to reproduce the jump at the critical temperature. An experimental check of Refs. [1] or [2] is proposed here. The study of the influence of the doping rate and the effects of a magnetic field is more interesting work in our opinion than to use the best band structure with two transfer integrals, as opposed to employing phenomenological parameters as we did. 


\section{References}

[1] J. Bok and J. Bouvier, Comment on paper [2].

[2] S. Dorbolo, M. Houssa and M. Ausloos, Physica C 267 (1996) 24.

[3] H.E. Stanley, Introduction to phase transition and critical phenomena, eds. W. Marshall and D.H. Wilkinson (Clarendon Press, Oxford, 1971).

[4] G. Rickayzen, Superconductivity, Vol. 1, ed. R.D. Parks (M. Dekker, New York, 1969) p. 79.
[5] L. Landau and E. Lifshitz, Physique Thérique, Tome 9, Physique Statistique, ed. Mir (CCCP, 1990).

[6] D.M. Newns, C.C. Tsuei and P.C. Pattnaik, Phys. Rev. B 52 (1996) 13611.

[7] D.M. Newns, C.C. Tsuei, P.C. Pattnaik and C.L. Kane, Commun. Condens. Matter Phys. 15 (1992) 273

[8] M.R. Norman, M. Randeria, H. Ding and J.C. Campuzano, Phys. Rev. B 52 (1995) 615.

[9] E. Dagotto, A. Nazarenko and A. Moreo, Phys. Rev. Lett. 74 (1995) 310. 